\title{
Lhermitte-Duclos Disease Mimicking Cerebellopontine Angle Tumor: A Case Report
}

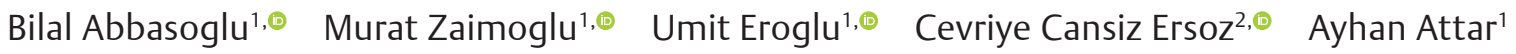

${ }^{1}$ Medical Faculty Brain and Nerve Surgery Department, Ankara University, Ankara, Turkey

${ }^{2}$ Medical Faculty Pathology Department, Ankara University, Ankara, Turkey

\begin{abstract}
Address for correspondence Bilal Abbasoğlu, MD, Department of Neurosurgery, Ankara University of Medical Faculty, Cebeci, Ankara 06590, Turkey (e-mail: bilalsukru@gmail.com).
\end{abstract}

Indian J Neurosurg 2021;10:169-172.

\begin{abstract}
Keywords

- Cerebellopontine angle

- Cerebellar gangliocytoma

- Lhermitte-Duclos disease

Lhermitte-Duclos disease (LDD) is dysplastic gangliocytoma of the cerebellum, which is rare, tends to grow slowly, usually has good prognosis, and wherein the cerebellar cortex becomes thick and the central white matter is not observed. On MRI of the brain, it has hyperintense and hypointense presentations on T2- and T1-weighted scans, respectively. It has nonhomogenous contrasting pattern on T1-contrast scans. LDD can be seen unilaterally or bilaterally in the cerebellum. When the disease is located at the cerebellopontine angle, it can be mistaken for the tumors located in this region. Recurrence can occur postoperatively, which is rare. The present case is a rare one because the tumor was located at the cerebellopontine angle and radiologically mimicked cerebellopontine angle tumors.
\end{abstract}

\section{Introduction}

Lhermitte-Duclos disease (LDD) is a benign hamartomatosis lesion of the cerebellum that tends to grow slowly. ${ }^{1}$ LDD is generally located in the cerebellar hemispheres and vermis. ${ }^{2,3}$ LDD can occur at any age; however, it is frequently diagnosed in people aged 20 to 40 years. ${ }^{4.5}$ The most common symptoms are headache, dizziness, nausea, vomiting, loss of balance, and impaired vision. ${ }^{6}$ When the tumor is located at the cerebellopontine angle, it can be radiologically mistaken for tumors that originate from this region. About 70\% to $90 \%$ tumors located in this region are vestibular schwannoma, whereas $10 \%$ of them are meningiomas. To date, no study has reported LDD to be located at the cerebellopontine angle. In the present case, the tumor is located at the cerebellopontine angle and mimics the tumors that originate from this region.

\section{Clinical Report}

A 29-year-old female patient visited our clinic with complaints of headache and dizziness. Physical examination showed normal results and neurological examination did not reveal any pathology except for horizontal nystagmus that became severe when the eyes moved to the left side. Cranial MRI revealed left cerebellopontine angle mass lesion that was nonhomogenous contrast-pattern on contrast T1-weighted scans ( - Fig. 1A-C); therefore, surgical treatment was planned for the patient. Left paramedian suboccipital craniectomy was performed as well as total excision of well-circumscribed tumor located at the left cerebellopontine angle. The patient was diagnosed with LDD based on the microscopic examination performed on the excised lesion (-Fig. 2). published online July 15, 2021
DOI https://doi.org/ 10.1055/s-0041-1725208 ISSN 2277-954X. (c) 2021. Neurological Surgeons' Society of India.

This is an open access article published by Thieme under the terms of the Creative Commons Attribution-NonDerivative-NonCommercial-License, permitting copying and reproduction so long as the original work is given appropriate credit. Contents may not be used for commercial purposes, or adapted, remixed, transformed or built upon. (https://creativecommons.org/licenses/by-nc-nd/4.0/).

Thieme Medical and Scientific Publishers Pvt. Ltd. A-12, 2nd Floor, Sector 2, Noida-201301 UP, India 

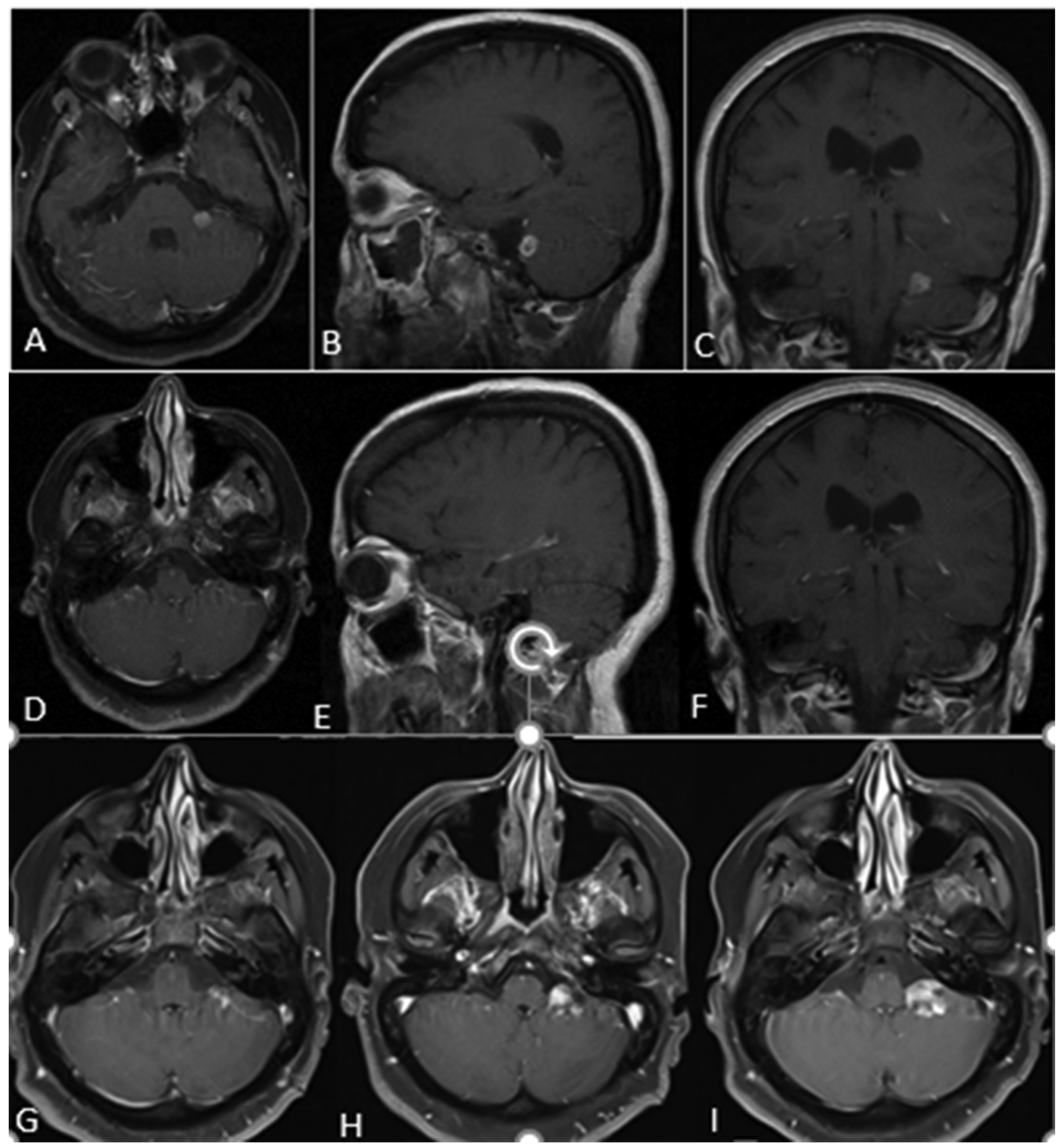

Fig. 1 (A) Preoperative contrast T1-weighed axial MR image; (B) Preoperative contrast T1-weighed sagittal MR image and (C) Preoperative contrast T1-weighed coronal MR image. No tumor detected on postoperative 1st-year follow-up MR images; (D-F). Gradually increasing tumor sizes on contrast T1-weighed axial MR images of the postoperative 2nd-, 3rd- and 4th-year (G-I).

The patient was followed up with annual cranial MRI, and the 1-year follow-up images did not reveal any newly-developed mass lesion ( - Fig. 1D-F). However, the 2nd, 3rd and 4th-year follow-up images gradually increasing revealed a mass at the operation site at the left cerebellopontine angle, which was nonhomogenous contrasting pattern on T1-weighted contrast scan (-Fig. 1G-I), but it did not cause any clinical symptom in the patient. Follow-up procedures revealed that the patient started to suffer from headache and dizziness. Therefore, surgery was planned for the patient. Almost complete excision of the tumor could be achieved by re-exploration. The clinical symptoms improved postoperatively.

\section{Discussion}

"Lhermitte-Duclos" in the 2016 Tumour Classification of the World Health Organisation is described as grade I benign 


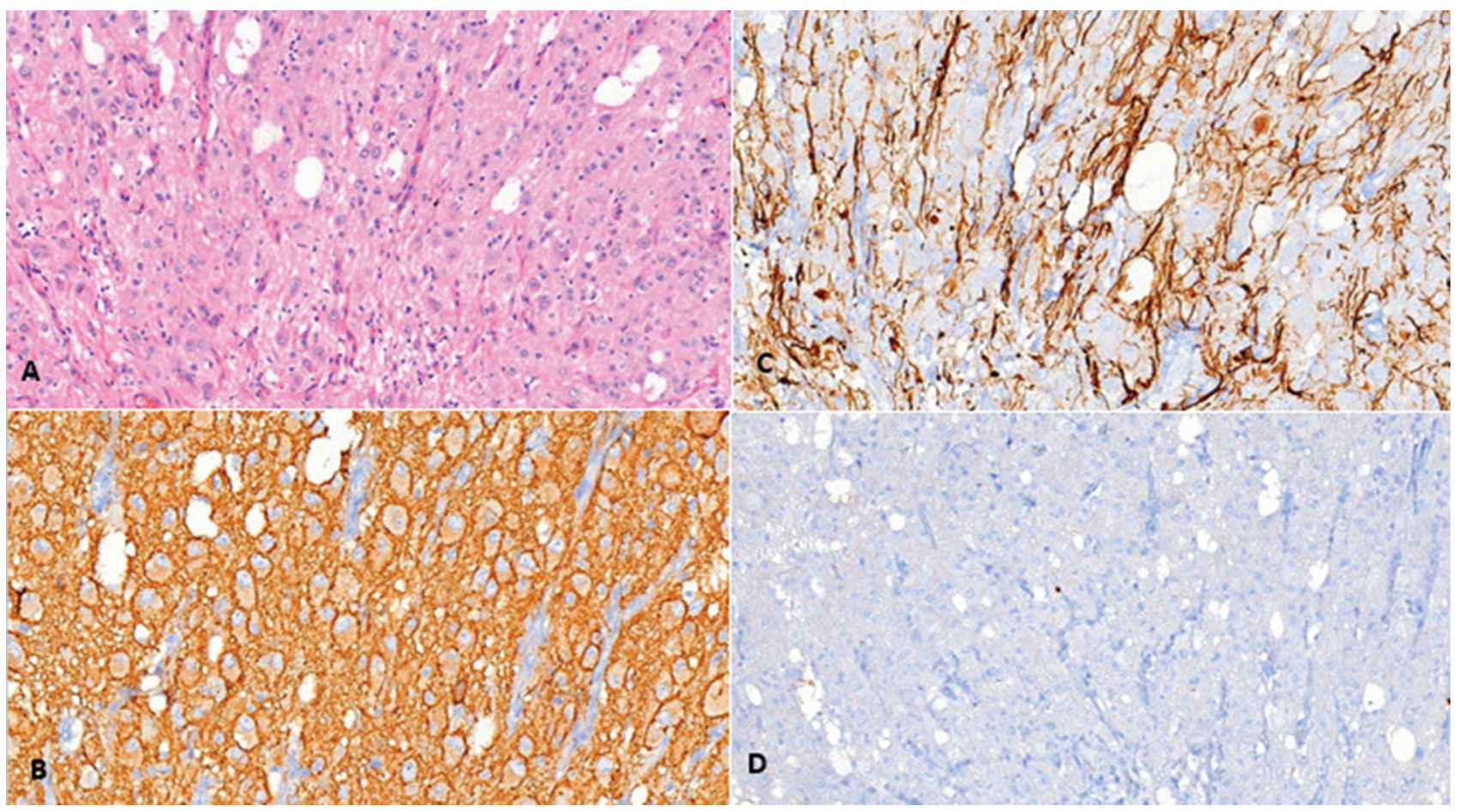

Fig. 2 (A) Large neuronal cells with irregularly located cells filling the internal granular layer, with large vesicle nucleus and large cytoplasm. Clear presence of vacuoles; H-Ex17.5; (B) Large hyperplastic cells filling the internal granular layer show membranous and slightly cytoplasmic staining with synaptophysin, which is a immunohistochemical neuronal indicator; SYNPx30.4; (C) Positively stained axons among the hyperplastic neuronal cells on immunohistochemical neurofilament protein (NFP) staining; NFPx21.4 and (D) Very low Ki-67 staining index in the large hyperplastic cells filling the internal granular layer; Ki67x16.2.

tumor. ${ }^{78}$ Although typically located unilaterally, there are also cases that have bilateral invasion. ${ }^{4}$ Accompanied with megalencephaly, syringomyelia, polydactyly, cranial asymmetry and mucocutaneous lesions, LDD is presented with Cowden syndrome that is characterized by autosomal dominantly-inherited hereditary hamartoma and neoplasia. ${ }^{9}$ Our patient did not have a history of cancer and skin lesions. LDD diagnosis is characterized by a tiger-striped pattern caused by abnormal cerebellar thickening that is hyperintense and hypointense on T2- and T1-weighted scans of cranial MRI, respectively. This pattern was not noted in our case. There are reported cases of contract-enhanced images similar to our case, although LDD does not present any obvious contrasting pattern. ${ }^{2,3}$ For the differential diagnosis of the cases with tumors located in the cerebellum, medulloblastoma, astrocytoma and cerebellar infarction should be considered. ${ }^{10}$ Because the tumor was located at the cerebellopontine angle in our case, the tumors located in this region should be considered for the differential diagnosis. The tumors located at the cerebellopontine angle are as follows: epidermoid tumors, arachnoid cysts, lipomas, metastases, lymphoma, paraganglioma, and hemangioblastoma. Moreover, brain stem tumors and glial tumors of the cerebellum have been reported to extend up to this region..$^{10}$

The most dominant treatment option is the "watchful waiting" strategy; however, surgery becomes important because of headache, nausea, vomiting, and hydrocephalia, which are associated with the pressure effect. ${ }^{4}$ As in our case, recurrence is observed postoperatively, although it is rare and benign. ${ }^{9}$ In addition, there are cases that do not present any clinical findings and are followed-up without surgery. 2,6

In our case, the location of the lesion at the cerebellopontine angle, which is very rare, led to the consideration of the pathologies of this region. However, following microscopic examination and literature review, it was understood that the tumor was associated with LDD, and it had an atypical pattern in terms of its location site, contrast-enhancing pattern and recurrence that occurred postoperatively.

\section{Conclusion}

Our case is informative and highlights the following: 1) LDD can have homogenous contrast-enhancing patterns, although rare; 2 ) there can be postoperative recurrences, although rare and 3) it can be present in the form of angle tumors along with the typical cerebellar hemispheric enhancement.

\section{Funding}

The pathological sections and images included in the present case study are supported by the Scientific Research Project (14A0230003).

\section{Conflict of Interest}

None declared. 


\section{References}

1 Ma J, Jia G, Chen S, Jia W. Clinical perspective on dysplastic gangliocytoma of the cerebellum (Lhermitte-Duclos disease) World Neurosurg 2019;122:16-23

2 Jain N, Chauhan U, Goel V, Puri SK. Lhermitte-Duclos disease: diagnosis on MRI, MR spectroscopy, CT and positron emission tomography. J Clin Diagn Res 2015;9(9):TJ01-TJ02

3 Cheng CS, Ou CH, Chen JS, Lui CC, Yeh LR. Lhermitte-Duclos disease: a case report with radiologic-pathologic correlation. Radiol Case Rep 2019;14(6):734-739

4 Borni M, Kammoun B, Kolsi F, Abdelmouleh S, Boudawara MZ. The Lhermitte-Duclos disease: a rare bilateral cerebellar location of a rare pathology. Pan Afr Med J 2019;33:118

5 Ezgu MC, Ozer MI, Dogan A, Deveci G, Kural C, Izci Y. Lhermitte-Duclos disease in a six-year old child: a rare presentation. Pediatr Neurosurg 2018;53(6):416-420
6 Wang Q Zhang S, Cheng J, Liu W, Hui X. Lhermitte-Duclos disease: Clinical study with long-term follow-up in a single institution. Clin Neurol Neurosurg 2017;162:53-58

7 Wesseling P, Capper D. WHO 2016 Classification of gliomas. Neuropathol Appl Neurobiol 2018;44(2):139-150

8 Önder E, Arikök AT, Türkoğlu E, Alper M. Lhermitte-Duclos disease: a rare lesion with variable presentations and obscure histopathology. Turk Patoloji Derg 2018;34(1):92-99

9 Khandpur U, Huntoon K, Smith-Cohn M, Shaw A, Elder JB. Bilateral recurrent dysplastic cerebellar gangliocytoma (Lhermitte-Duclos disease) in Cowden syndrome: a case report and literature review. World Neurosurg 2019;127:319-325

10 Friedmann DR, Grobelny B, Golfinos JG. Roland JT Jr. Nonschwannoma tumors of the cerebellopontine angle. Otolaryngol Clin North Am 2015;48(3):461-475 